\section{Dengue y dengue hemorrágico en las Américas}

\author{
Mirta Roses Periago y \\ María G. Guzmán²
}

A pesar de que el dengue se conoce como entidad clínica desde hace más de dos siglos y del conocimiento acumulado en el transcurso de los últimos años, esta arbovirosis continúa siendo hoy uno de los principales problemas de salud mundial y constituye uno de los mayores retos de salud pública en el milenio actual.

La infección, causada por cualquiera de los cuatro serotipos del virus del dengue y transmitida al hombre por la picada del mosquito Aedes aegypti, su principal vector, puede cursar en forma asintomática, producir el cuadro clásico de la enfermedad (dengue) — de evolución benigna- o el cuadro grave y a menudo fatal (dengue hemorrágico/síndrome de choque del dengue, $\mathrm{DH} / \mathrm{SCD}$ ), caracterizado por el incremento en la permeabilidad vascular, hemorragias, hemoconcentración y trombocitopenia.

Teniendo en cuenta la emergencia y reemergencia de esta enfermedad y la grave situación epidemiológica imperante en la Región de las Américas — que además de afectarnos a todos constituye una nefasta herencia que dejaremos a las generaciones futuras si no actuamos rápida, inteligente e integradamente-, la Revista Panamericana de Salud Pública/Pan American Journal of Public Health (RPSP/PAJPH) publicó durante el último año una serie de artículos sobre el dengue con el objetivo de llamar la atención de los lectores sobre este tema y de apoyar los esfuerzos que se realizan para combatir esta enfermedad. En esta serie se presentaron artículos de investigación y de discusión sobre los más disímiles aspectos, todos de la mayor importancia.

En el editorial que dio inicio a la serie sistemática, "El dengue, un problema creciente de salud en las Américas", Gustavo Kourí analizó la situación epidemiológica mundial del dengue y la carga que representa esta enfermedad (1). Según la Organización Mundial de la Salud (OMS), más de 100 países —en los que viven 2500 millones de personas- informan de casos de dengue, de ellos más de 500000 son de DH/SCD y alrededor de 25000 personas fallecen por esa enfermedad anualmente. En este editorial se destacan los cuatro elementos básicos para controlar el dengue: la voluntad política, la coordinación intersectorial, la participación activa de la comunidad y el fortalecimiento de las leyes sanitarias.

Este análisis se complementa con el examen de las prioridades de investigación a corto y mediano plazos, presentado por Guzmán y colaboradores, basado en la información científica más actualizada y la situación epidemiológica mundial, particularmente en las Américas (2). Nuestra Región es un ejemplo de la emergencia y la reemergencia del dengue. La expansión de la circulación del virus del dengue tipo 2 en la década de 1950, la aparición del serotipo 3 en la década de 1960, del serotipo 1 en la de 1970 y del serotipo 4 en la de 1980, así como las primeras pandemias ocurridas en los años 1962-1963, 1968-1969, 19771978 y la detección de casos de DH/SCD en Cuba en 1981 y posteriormente en muchos otros países del área crearon las bases para la situación actual. La reinfestación paulatina durante las décadas de 1970 y 1980 de países que estaban libres del vector y la entrada de genotipos virales de mayor virulencia han complicado aun más el cuadro epidemiológico de la Región.

Para enfrentar esta grave situación, la Organización Panamericana de la Salud (OPS) ha desarrollado la Estrategia de Gestión Integrada para la Prevención y el Control del Dengue (EGI-dengue) en la Región de las Américas, analizada por San Martín y Brathwaite-Dick en el número de la RPSP/PAJPH correspondiente a enero de 2007 (3). En este artículo se hace una pormenorizada descripción de los objetivos, la puesta en marcha y los resultados preliminares de la EGI en los 11 países en que se ha implementado. Para ello, en cada país se analizan las condiciones específicas en cinco áreas vitales: la comunicación social, la vigilancia epidemiológica, el diagnóstico de laboratorio, la atención al
Directora, Oficina Sanitaria Panamericana, Washington, D.C., Estados Unidos de América. Instituto de Medicina Tropical Pedro Kourí, Ciudad de La Habana, Cuba. 
paciente, y el control del vector y el cuidado del medio ambiente. Se espera que la generalización de esta estrategia permita controlar la transmisión y reducir la incidencia de esta enfermedad mediante un enfoque integrado e intersectorial.

La reducción de la mortalidad por dengue, otra de las prioridades de los sistemas nacionales de salud, es el objeto del artículo "La prevención de la mortalidad por dengue: un espacio y un reto para la atención primaria de salud"(4). En este artículo, Eric Martínez se basa en su amplia experiencia y la información disponible más actualizada para analizar y desentrañar los mitos y los errores que provocan una mayor mortalidad por esta enfermedad y se hace hincapié en la necesidad de identificar tempranamente los signos clínicos de alarma que permiten aplicar más oportunamente el tratamiento establecido y evitar el desarrollo de las formas más graves, como el DH/SCD. Basado en la experiencia de cuatro países (Brasil, Cuba, El Salvador y Tailandia) se concluye que la calidad de la atención médica, el acceso oportuno de la población a los servicios de salud, la prevención, y la promoción y educación sanitaria de la comunidad son los aspectos fundamentales para reducir la mortalidad por dengue. En cuanto a la formación del personal de salud, el adiestramiento de los médicos y paramédicos en la aplicación de una clasificación clínica sencilla y dinámica y el establecimiento de normas terapéuticas adecuadas y acordes a cada etapa de la enfermedad constituyen pilares fundamentales para la reducción de la mortalidad. Como complemento, pero no de menor importancia, se destaca la necesidad de educar a la población, reordenar los servicios y garantizar los recursos médicos necesarios. La adecuada información de la población permite que se puedan identificar más rápidamente los síntomas de la enfermedad, que la familia y la comunidad participen en su autocuidado correctamente y que se soliciten más tempranamente los servicios de atención médica. Por su parte, el reordenamiento del sistema de atención médica para mejorar el diagnóstico y el tratamiento de todos los casos, así como la observación del paciente grave, deben también incidir positivamente en la disminución de la mortalidad (4).

Un tema de especial interés por sus implicaciones en la comprensión de la epidemiología es el relacionado con el estudio de los mecanismos patogénicos del virus del dengue y el desarrollo de vacunas y medicamentos para su prevención y tratamiento. Aunque la patogenia del dengue no ha sido completamente aclarada, se conoce que la infección secundaria por un serotipo diferente al que produce la infección primaria es uno de los principales factores de riesgo de las formas clínicas graves y constituye uno de los principales retos en el desarrollo de una vacuna contra el dengue. Una vacuna eficaz debe brindar inmunidad contra los cuatro serotipos virales y evitar el fenómeno de inmunoamplificación dependiente de anticuerpos. Estos aspectos, abordados por Guzmán y colaboradores (2), constituyen el preámbulo a un documentado artículo de Hombach que ofrece una muy actualizada revisión sobre las vacunas contra el dengue (5).

Según explica Hombach (5), en la actualidad se cuenta con varias vacunas candidatas (recombinantes, vivas atenuadas y quiméricas de clones infecciosos) en diferentes fases de ensayos clínicos y preclínicos. Se espera que en los próximos cinco años se cuente con una vacuna aprobada contra el dengue que haya demostrado su eficacia en estudios multicéntricos realizados en diferentes regiones geográficas, especialmente en Asia y las Américas. En estos momentos se analiza cuáles pueden ser los mejores sitios para estos estudios, se definen los correlatos inmunológicos de protección y se evalúan las estrategias adecuadas de vacunación.

A pesar del conocimiento acumulado sobre la enfermedad y su patogenia y de los avances en el desarrollo de vacunas, el control del mosquito vector continúa siendo aún hoy la única herramienta eficaz para controlar la transmisión. El control vectorial sostenible representa todavía un gran reto para los países afectados. La identificación de los factores ecológicos que favorecen la infestación por A. aegypti y de los principales sitios de cría puede ayudar a implementar acciones de control más adecuadas y menos costosas. En un artículo sobre este tema, Bisset y colaboradores (6) informan que los tanques bajos y los pequeños depósitos artificiales destinados a almacenar agua presentaron el mayor riesgo de infestación 
por el vector en cuatro áreas de salud de la capital cubana en 2004-2005. Se observó, además, una elevada correlación entre el número de depósitos infestados ubicados en patios y la presencia de vegetación y árboles, la ubicación en lugares de sombra parcial o total y la deficiente higiene doméstica. De acuerdo con esos resultados, las acciones de control se deben dirigir a tres elementos fundamentales: 1) la educación y movilización de la comunidad en el tapado correcto de los tanques bajos y en la aplicación del insecticida recomendado; 2) la correcta disposición de los recipientes y envases inútiles o desechados para evitar su exposición a la lluvia; y 3) el recambio del agua en los bebederos de animales. Los autores concluyen que la participación activa de la población permite complementar los esfuerzos de las autoridades sanitarias en la lucha antivectorial, por lo que se recomienda informar adecuadamente a la población, vencer su apatía y crear una cultura comunitaria que promueva una mayor higiene ambiental.

Un aspecto poco conocido relacionado con el dengue - pero de la mayor importancia - es el impacto económico que la enfermedad impone a los países. Este es el tema tratado por Añez y colaboradores en su artículo "Impacto económico del dengue y del dengue hemorrágico en el Estado de Zulia, Venezuela, 1997-2003" (7). Después de analizar los costos directos e indirectos asociados con la atención de enfermos de dengue en ese estado venezolano, los autores concluyen que el costo promedio de hospitalización de un paciente con dengue hemorrágico durante una semana fue de US\$ 94,90 y comparan sus resultados con los informados en Cuba, Nicaragua y Puerto Rico.

En un muy documentado artículo (8), Scott Halstead analiza cómo la diversidad genética de las poblaciones asiáticas y americanas puede ser la base de las diferencias epidemiológicas observadas entre ambas regiones. Mientras en el sudeste asiático se notificaron 1,16 millones de casos de dengue hemorrágico - principalmente en niños- en un lapso de cinco años, en nuestra Región se informaron 2,8 millones de casos de dengue - principalmente en adultos- y solo 65000 casos de dengue hemorrágico. Esta información es crucial para elaborar estrategias apropiadas de lucha contra el dengue en los contextos nacional, regional y mundial.

Frente al agravamiento de la situación epidemiológica internacional, previsto para las próximas décadas, los países y las organizaciones internacionales implementan diferentes iniciativas, adaptadas a la situación local concreta, con el objetivo de prevenir y controlar las epidemias de dengue. Y la OPS está lista para cumplir sus compromisos.

La estrategia propuesta por la OMS en 1995 para frenar la transmisión del dengue (9), basada en la centralización y la coordinación de los esfuerzos nacionales, se vio reforzada en 2002 con la aprobación de una resolución sobre la prevención y control del dengue y del dengue hemorrágico, aprobada en la 55. ${ }^{\mathrm{a}}$ Asamblea Mundial de Salud (9). La estrategia internacional propuesta consta de cinco elementos fundamentales:

- el control selectivo e integrado del vector con la participación de la comunidad y con respaldo intersectorial

- la vigilancia activa de la enfermedad mediante el diagnóstico de laboratorio y la vigilancia entomológica

- la preparación de las condiciones para el enfrentamiento de las situaciones de emergencia

- el desarrollo de capacidades y el adiestramiento de los recursos humanos

- la investigación sobre métodos efectivos y eficaces de control del vector.

En la Región de las Américas, la respuesta fue inmediata. La resolución CD43.R4 aprobada en la 43. ${ }^{\text {a }}$ Reunión del Consejo Directivo de la OPS en 2002 (10) se constituyó en el marco de referencia para una nueva generación de programas de prevención y control del dengue. Estos programas se basarían en lo adelante en la vigilancia integrada, la abogacía e implementación de acciones intersectoriales - con la intervención de las autoridades nacionales de salud, 
medio ambiente y educación, entre otros-, la activa participación de la comunidad, la preservación del medio ambiente y el mejoramiento de los servicios básicos de abastecimiento de agua, de disposición de residuales y de gestión de neumáticos desechados. Otras de las medidas previstas son el fortalecimiento de la atención al paciente, el mejoramiento del sistema de notificación de casos y la incorporación del estudio del dengue a los currículos del sistema formal de educación. Además, se debe dar una correcta utilización a los insecticidas y mejorar la capacitación del personal y la preparación de los sistemas de salud para el enfrentamiento de las emergencias.

Un año después, durante la $44 .^{\text {a }}$ Reunión del Consejo Directivo de la OPS, esta estrategia tomó forma mediante la resolución CD44.R9, que estableció los métodos para implementar los procesos de cambio en los programas nacionales (11) mediante la implementación de las EGI-dengue nacionales y la creación de un grupo regional de trabajo técnico en dengue.

En los próximos años, la OPS continuará apoyando el proceso de fortalecimiento de las capacidades nacionales de prevención y control del dengue en los Estados Miembros mediante la extensión de la EGI-dengue. Se hará un esfuerzo especial en apoyar a las autoridades de los Estados Miembros en la implementación de esta estrategia y en la evaluación sistemática de ese proceso. A pesar de los avances observados en poco tiempo, todavía se deben modificar los factores determinantes de la salud que constituyen factores de riesgo en la transmisión del dengue, como la urbanización no controlada, la falta de servicios básicos de higienización y la pobreza.

Otro elemento importante que continuará contando con el apoyo de la OPS es la implementación de la metodología de comunicación para lograr cambios de conducta (COMBI), que dará una mayor sostenibilidad a las acciones de prevención y control. Hasta el momento, 22 países han recibido capacitación en el uso de esta metodología y se implementan planes específicos dirigidos a lograr la adecuada manipulación y limpieza de los contenedores domésticos de agua, la disposición de los neumáticos inservibles y de desechos sólidos, y el aumento de la capacidad de reconocimiento de la enfermedad, tanto por el personal médico como por el mismo paciente y la comunidad. Según los resultados preliminares disponibles, estos planes han contribuido a fortalecer los programas nacionales mediante la participación activa de la comunidad y otros actores sociales.

El sinergismo logrado por la implementación de las EGI-dengue nacionales y los planes COMBI-dengue ha elevado sustancialmente el impacto positivo de los recursos dedicados a la lucha contra esta enfermedad, por lo que se espera que estas iniciativas contribuyan a reducir eficaz y eficientemente la morbilidad, la mortalidad y la carga social y económica causada por los brotes y epidemias de dengue.

La OPS continuará apoyando las investigaciones dirigidas a reducir la transmisión de la enfermedad y a mejorar la atención de los enfermos. Kroeger ha propuesto agrupar las prioridades de investigación en dengue en cuatro direcciones principales (12), todas pertinentes y de gran importancia para nuestra Región:

- reducir la gravedad de la enfermedad y la mortalidad asociada, con especial énfasis en la optimización y estandarización de los procedimientos de atención clínica del paciente, la capacitación del personal y la investigación de las bases genéticas, tanto del virus como del huésped, que influyen en la patogénesis de la enfermedad

- disponer de nuevas herramientas y estrategias para el control más eficaz del vector y una mejor vigilancia epidemiológica

- ampliar las medidas de prevención primaria y secundaria mediante el desarrollo y aplicación de vacunas eficaces y de nuevos medicamentos

- contar con políticas de salud que contribuyan a reducir los factores de riesgo en la transmisión del dengue y la evolución de la enfermedad a formas más graves. 
Como señalan Troyo y colaboradores en su artículo "Dengue en Costa Rica: la brecha en la investigación científica local" (13), la investigación científica puede contribuir a desarrollar nuevos enfoques y métodos de control. Está demostrada la necesidad de incrementar la colaboración interinstitucional en investigaciones, aumentar la preparación del personal, elevar la capacidad investigativa y estimular la publicación oportuna de los resultados.

No quedan dudas del peligro que representa el dengue para las regiones tropical y subtropical y de sus implicaciones para el mundo desarrollado. La expansión actual y perspectiva del turismo - y en consecuencia, la mayor posibilidad de transmisión de la enfermedad- exigen un mayor compromiso por parte de todos los países - ricos y pobres - y de las organizaciones internacionales. Es de suma importancia lograr una mayor comprensión del problema y de la necesidad de su enfrentamiento global.

La situación actual en nuestra Región es compleja, no obstante existen las bases para detener la tendencia creciente del dengue y revertirla. La mayoría de los países cuentan con programas de prevención y control -aunque con diferentes grados de desarrollo-, existe una red de laboratorios con capacidad diagnóstica y se cuenta con varios centros colaboradores de la OPS, cuya gestión puede y debe tener un mayor impacto en el enfrentamiento del dengue y de otros agentes patógenos, mediante un trabajo coordinado. Finalmente - y de la mayor importancia-, el actual fortalecimiento de los sistemas de salud pública en varios de los países de la Región y la creciente voluntad política son factores decisivos para lograr la integración, la intersectorialidad y la prioridad necesarias para enfrentar al dengue. Las condiciones creadas y la estrategia establecida pueden y deben permitir alcanzar la meta de detener la expansión creciente del dengue en la Región y progresivamente comenzar a reducir la morbilidad, la mortalidad y el impacto social y económico de la enfermedad en las Américas.

\section{REFERENCIAS}

1. Kourí G. El dengue, un problema creciente de salud en las Américas. Rev Panam Salud Publica. 2006;19(3):143-5.

2. Guzmán MG, García G, Kourí G. El dengue y el dengue hemorrágico: prioridades de investigación. Rev Panam Salud Publica. 2006;19(3):204-15.

3. San Martín JL, Brathwaite-Dick O. La Estrategia de Gestión Integrada para la Prevención y el Control del Dengue en la Región de las Américas. Rev Panam Salud Publica. 2007; 21(1):55-63.

4. Martínez E. La prevención de la mortalidad por dengue. Rev Panam Salud Publica. 2006; 20(1):60-74.

5. Hombach J. Vaccines against dengue. Rev Panam Salud Publica. 2007;21(4):254-60.

6. Bisset JA, Marquetti MC, Portillo R, Rodríguez MM, Suárez S, Leyva M. Factores ecológicos asociados con la presencia de larvas de A. aegypti en zonas de alta infestación del municipio Playa, Ciudad de La Habana, Cuba. Rev Panam Salud Publica. 2006;19(6):379-84.

7. Añez G, Balza R, Valero N, Larreal Y. Impacto económico del dengue y del dengue hemorrágico en el estado de Zulia, Venezuela, 1997-2003. Rev Panam Salud Publica. 2006; 19(5):314-20.

8. Halstead SB. Dengue in the Americas and Southeast Asia: do they differ? Rev Panam Salud Publica. 2006;20(6):407-15.

9. World Health Organization. Strengthening implementation of the global strategy for dengue fever/dengue haemorrhagic fever prevention and control. Report of the Informal Consultation. Geneva: WHO; 1999. (WHO/CDS/DEN (IC)/2000.1).

10. Organización Panamericana de la Salud. Marco de referencia. Nueva generación de programas de prevención y control del dengue en las Américas. Washington, D.C.: OPS; 2002. (OPS/HCP/HCT/206/02).

11. Pan American Health Organization. 44th Directing Council. Resolution CD44.R9. Dengue. Washington, D.C.: PAHO; 2003. Hallado en: http://www.paho.org/english/gov/cd/cd44r9-e.pdf. Acceso el 8 de marzo de 2007.

12. Kroeger A, Nathan M. Dengue: setting the global research agenda. Lancet. 2006;368:2193-5.

13. Troyo A, Porcelain SL, Calderón-Arguedas O, Chadee DD, Beier JC. Dengue en Costa Rica: la brecha en la investigación científica local. Rev Panam Salud Publica. 2006;20(5):350-60. 